STRUCTURAL BIOLOGY
Received 14 August 2018

Accepted 25 January 2019

Keywords: protein; simulation; phasing; multi-microcrystallography; radiation damage.

Supporting information: this article has supporting information at journals.iucr.org/d

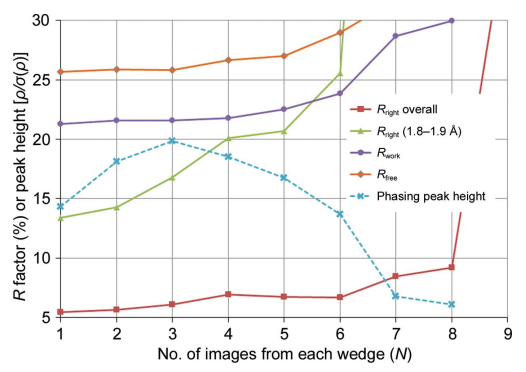

OPEN $\odot$ ACCESS

\section{Challenge data set for macromolecular multi-microcrystallography}

\author{
James M. Holton*
}

Department of Biochemistry and Biophysics, University of California, San Francisco, CA 94158-2330, USA, Divison of Molecular Biophysics and Bioengineering, Lawrence Berkeley National Laboratory, Berkeley, CA 94720, USA, and Stanford Synchrotron Radiation Lightsource, SLAC National Accelerator Laboratory, Menlo Park, CA 94025, USA. *Correspondence e-mail: jmholton@|bl.gov

A synthetic data set demonstrating a particularly challenging case of indexing ambiguity in the context of radiation damage was generated. This set shall serve as a standard benchmark and reference point for the ongoing development of new methods and new approaches to robust structure solution when singlecrystal methods are insufficient. Of the 100 short wedges of data, only the first 36 are currently necessary to solve the structure by 'cheating', or using the correct reference structure as a guide. The total wall-clock time and number of crystals required to solve the structure without cheating is proposed as a metric for the efficacy and efficiency of a given multi-crystal automation pipeline.

\section{Introduction}

Data sets that challenge the capabilities of modern structuresolution procedures, algorithms and software are difficult for developers to obtain for a very simple reason: as soon as a solution is reached, the data set is no longer considered to be challenging. Data sets that are recalcitrant to current approaches are also not available in public databases such as the Protein Data Bank (Berman et al., 2002) or image repositories (Grabowski et al., 2016; Morin et al., 2013) that only contain data used for solved structures. When testing the limits of software, it is generally much more useful to know ahead of time what the correct result will be. This enables the detection and optimization of partially successful solutions at every point in the process, even if downstream procedures fail.

There is a fundamental limit to how small a protein crystal can be and still yield a complete data set (Holton \& Frankel, 2010), so as beams and crystals become smaller and smaller the use of multi-crystal data sets becomes unavoidable. The purpose of the challenge presented here was to represent a situation in which the user decided to take relatively long exposures for each image in order to ensure that the highresolution spots were visible to the eye. For small crystals, however, much of the useful life of the sample is used up in the first few images using this strategy (Evans et al., 2011), and the challenge is to reassemble all of the data from a large number of highly incomplete data-collection runs, or wedges.

A low-dose reference data set could greatly reduce the challenges presented here, but only because this is a case of high isomorphism. Real crystals always have some sample-tosample variability, and may even have more than one crystal habit. Multiple habits are often related by pseudo-symmetry, making it very difficult to distinguish between genuinely heteromorphic crystals and variable indexing software performance. In such cases, which crystal to use as a reference is in no way obvious. Enforcing a presumed unit cell and space 
group increases the indexing hit rate, but will make the final data worse if intensities are merged from incompatible crystals. For this reason, the present challenge was posed without a reference, and perfect isomorphism was employed only to aid in scoring the results.

\section{Methods}

\subsection{Preparation of simulated structure factors $\left(\boldsymbol{F}_{\text {right }}\right)$}

Although it is possible to input $F_{\text {obs }}$ data into a MLFSOM (Holton et al., 2014) simulation, $F_{\text {obs }}$ is seldom $100 \%$ complete, and any missing $h k l$ s provided to MLFSOM will be taken as zero when rendering the simulated images, and thus imageprocessing software will assign them a well measured intensity of zero. This will happen even if the reason for the missing $F_{\text {obs }}$ was because the spot was saturating the detector in the original experiment, which is a very large and unnatural systematic error. In addition, the anomalous differences of $F_{\text {obs }}$ are invariably noisy, and are often unavailable. For these reasons, it is convenient to use calculated structure factors, which are always $100 \%$ complete, have a well known phase and, by definition, no error in the amplitudes. Additional systematic errors can then be clearly defined and applied, depending on the goals of the simulation.

Calculated structure factors such as those output from refinement programs are typically denoted $F_{\text {calc }}$, but for clarity here $F_{\text {right }}$ shall denote the calculated structure factors that are fed into an image simulator. Thus, $F_{\text {right }}$ denotes the 'right answer' used to evaluate the data-processing results. Structure factors obtained from simulated images shall be denoted $F_{\text {sim }}$, as opposed to $F_{\text {obs, }}$, which will be reserved for actual real-world experimental observations. The distinction is important because the dominant source of systematic error in macromolecular crystallography that leads to the characteristically large ' $R$-factor gap' between $F_{\text {obs }}$ and $F_{\text {calc }}$ is much larger than all experimental measurement errors combined (Holton et al., 2014), but the exact nature of this source of error remains unclear. Specifically, refinement against $F_{\text {right }}$ or $F_{\text {sim }}$ derived from a simple single-conformer model invariably converges to abnormally low $R_{\text {work }}$ and $R_{\text {free }}$ after automated building and refinement. This is a glaring inconsistency with real data, and potentially makes the simulated data unrealistically easy to solve, diminishing their usefulness in benchmarking and debugging. More realistic $R$ factors can be obtained by adding random numbers to $F_{\text {right }}$, but the appropriate random distribution to use is not clear. Instead, values of $F_{\text {right }}$ were generated here to have a combination of physically plausible systematic errors and one final empirical systematic error.

\subsection{I1 domain from titin (PDB entry 1g1c): lysozyme's evil} twin

The titin I1 domain was selected because the PDB entry $1 \mathrm{~g} 1 \mathrm{c}$ (Mayans et al., 2001), with unit-cell parameters $a=38.3$, $b=78.6, c=79.6 \AA$, is the closest nontetragonal unit cell to that of tetragonal Gallus gallus egg lysozyme. The true space group is $P 2_{1} 2_{1} 2_{1}$, and thus represents an excellent challenge to software developers seeking to resolve indexing ambiguity in multi-crystal projects, automatic space-group assignment, detection of non-isomorphism from cell variation (Foadi et al., 2013) and identification of crystallization contaminants by searching for similar unit cells in a database (McGill et al., 2014; Simpkin et al., 2018).

Coordinates and observed structure-factor data for entry $1 \mathrm{~g} 1 \mathrm{c}$ were downloaded from the PDB (Berman et al., 2002) and the CIF-formatted structure-factor data were converted to MTZ format using the $C I F 2 M T Z$ program from the $C C P 4$ suite (Winn, 2003). The MTZ file header was edited with MTZUTILS to make $a=38.3 \AA$ and $b=c=79.1 \AA$. The deposited coordinates were then refined against the new MTZ file using phenix.refine (Adams et al., 2010) for three macrocycles.

This single-conformer model was used to compute $F_{\text {right }}$ for a preliminary MLFSOM simulation, but downstream analysis suffered from the unrealistically low $R_{\text {free }}<2 \%$ statistics mentioned above. Previous studies (Holton et al., 2014) found that using $F_{\text {right }}$ from a multi-conformer model leads to a more realistic $R_{\text {free }}$, but modern building programs such as qFit (van den Bedem et al., 2009) can easily identify two or three alternate conformations. Real crystals contain trillions of different conformations, but approximating them as a Gaussian distribution simply recovers a canonical $B$ factor. Therefore, in order to create physically plausible systematic error that is not easily captured by automated building, twenty alternate conformations were generated for this simulation.

Twenty new PDB files were created from the singleconformer reference by perturbing each atom position, including all waters, with a random coordinate shift consistent with the assigned atomic $B$ factor $\left(B_{\text {atom }}\right)$ using the jigglepdb.awk script distributed with MLFSOM (Holton et al., 2014). Each of the twenty perturbed models was then refined against the re-indexed $F_{\text {obs }}$ data using phenix.refine (Adams et al., 2010) for ten macrocycles with no free- $R$ flags. This operation allowed the coordinates to relax away from any clashes and geometric distortions owing to the unit-cell change and random coordinate shifts and at the same time become more consistent with $F_{\text {obs }}$. The reason for disabling the free- $R$ flags was to avoid creating an artificial $R_{\text {work }}$ versus $R_{\text {free }}$ bias in $F_{\text {right. }}$.

The algorithm in the jigglepdb.awk program simply shifts each atom along $x, y$ and $z$ using three independent Gaussian deviates taken from a distribution with root-mean-square (r.m.s.) variation equal to $\left(B_{\text {atom }} / 24\right)^{1 / 2} / \pi$. This is the r.m.s. shift that recapitulates the $B$ factor at infinite trials. For example, consider a C atom with $B_{\text {atom }}=5 \AA^{2}$ versus $B_{\text {atom }}=29 \AA^{2}$. The electron density of both of these cases is readily available using standard crystallography software such as SFALL (Winn, 2003) or phenix.fmodel (Adams et al., 2010), but let us suppose that only $B_{\text {atom }}=5 \AA^{2}$ is available and we want $B_{\text {atom }}=29 \AA^{2}$. In that case we must 'simulate' an additional $B$ factor of $24 \AA^{2}$ by calculating and averaging millions of maps with $B_{\text {atom }}=5 \AA^{2}$, each after randomly shifting the atom from its starting point. If the r.m.s. shift in any given direction is $0.318 \AA$, we obtain a map identical to what we would have 
obtained with $B_{\text {atom }}=29 \AA^{2}$. This is because an r.m.s. shift of $0.318 \AA$ corresponds to $B=24 \AA^{2}$ and $B$ factors are additive $(5+24=29)$. Therefore, atomic shifts of $\left(B_{\text {atom }} / 24\right)^{1 / 2} / \pi$ represent the natural deviations that are expected to be found from unit cell to unit cell in the crystal.

The final r.m.s. deviations between these twenty re-refined models ranged from 0.75 to $0.9 \AA\left(0.27-0.34 \AA\right.$ for $\mathrm{C}^{\alpha}$ atoms only). Each re-refined model was then edited to change all four methionine $\mathrm{S}$ atoms to selenium. The refined solvent parameters $k_{\text {sol }}, B_{\text {sol }}, R_{\text {solv }}$ and $R_{\text {shrink }}$ were extracted from each phenix.refine run and then used with the selenium-containing coordinates in phenix.fmodel to generate twenty complete sets of calculated anomalous structure factors $\left(F_{\text {model }}\right)$ out to $1.8 \AA$ resolution. These twenty $F_{\text {model }}$ sets differed from each other by $14-20 \%$, and were combined together into a single amplitude $F_{\text {r.m.s. }}$ by taking the square root of the mean-square $F_{\text {model }}$,

$$
\left|F_{\text {r.m.s. }}\right|=\left\langle\left|F_{\text {model }}\right|^{2}\right\rangle^{1 / 2},
$$

where $\|$ denotes the amplitude and \langle\rangle the average value. Note that $F_{\text {r.m.s. }}$ is not an error estimate; it is simply an intensitydomain average of the twenty $F_{\text {model }}$ amplitudes. $F_{\text {r.m.s. }}$ is not equivalent to averaging the electron-density maps $\left(F_{\text {avg }}\right)$, which is mathematically identical to averaging $F_{\text {model }}$ as complex numbers. The difference is that $F_{\text {avg }}$ assumes that all twenty structures can be found within the coherence length of the beam, whereas $F_{\text {r.m.s. }}$ represents the assumption that the twenty structures make up twenty different types of independently diffracting mosaic domains. The $R$ factor between $F_{\text {avg }}$ and $F_{\text {r.m.s. }}$ was only $3.3 \%$, but since $F_{\text {r.m.s. }}$ represents a physically plausible systematic error, it was carried on to the next step.

An empirical ' $R$-factor gap' systematic error was extracted by refining the deposited $1 \mathrm{~g} 1 \mathrm{c}$ model against the deposited $1 \mathrm{~g} 1 \mathrm{c}$ data and taking the $F_{\text {obs }}-F_{\text {calc }}$ amplitude difference for all observed reflections $\left(F_{\text {diff }}\right) . F_{\text {diff }}$ was taken to be an empirical systematic error and added to $F_{\text {r.m.s. }}$ to form $F_{\text {sys. }}$ Reflections missing $F_{\mathrm{obs}}$ were given $F_{\text {diff }}=0$, and the resulting $R$ factor between $F_{\text {r.m.s. }}$ and $F_{\text {sys }}$ was $18 \%$. Finally, the resolution was made to be slightly better than that available in PDB entry $1 \mathrm{~g} 1 \mathrm{c}$ with a sharpening filter. This was performed by applying a $B$ factor of $-15 \AA^{2}$ to $F_{\text {sys }}$ to form the value of $F_{\text {right }}$ that was fed into the MLFSOM (Holton et al., 2014) simulation.

\subsection{Image-simulation runs}

Image simulations were conducted with MLFSOM (Holton et al., 2014) using parameters matching the behavior of an Area Detector Systems (ADSC; Poway, California, USA) model Q315r X-ray detector, which is essentially a powdered $\mathrm{Gd}_{2} \mathrm{O}_{2} \mathrm{~S}$ phosphor bonded to a charge-coupled device (CCD) via a fiber-optic taper (Holton et al., 2012; Gruner et al., 2002; Gruner, 1989; Waterman \& Evans, 2010). These parameters were an electro-optical gain of 7.3 CCD electrons per X-ray photon, an amplifier gain of 4 electrons per pixel intensity unit (ADU), a zero-photon pixel level or 'ADC offset' set to 40
ADU, and a readout noise of 16.5 electrons r.m.s. per pixel. An intensity vignette falling to $40 \%$ at the edge of each module was used, and the Moffat function for the fiber-coupled CCD point-spread function, as described in Holton et al. (2012), was varied from a $g$ value of $30 \mu \mathrm{m}$ at the center of each module to $60 \mu \mathrm{m}$ at the corner. The calibration error was set to $3 \%$ r.m.s. with a spatial period of 50 pixels. This is in contrast to the true detector behavior of subpixel calibration error (Waterman \& Evans, 2010), but had been found in previous simulations to produce realistic $R_{\text {merge }}$ values.

Image header values were made to be exact, with the exception of the beam center, which always requires further qualification. The header value was $x, y=154.96,155.7$, which is one pixel off in each direction from the true beam center $(155.063,155.647)$ in the convention of the $A D X V$ diffraction-image viewer program (Szebenyi et al., 1997; Arvai, 2012). This one-pixel shift is an example of the unfortunately common array of caveats that can enter into a beam center. Switching between programs that start counting pixels at 1 versus 0 will generate one-pixel shifts, and changing the definition of a pixel location from its center to one of the corners results in half-pixel shifts. More serious changes in beamcenter convention involve swapping the $x$ and $y$ axes, changing the origin among the four corners of the image and two possible mirror flips. Different processing programs have different conventions and, despite significant efforts to standardize them (Parkhurst et al., 2014), do not always recognize and convert header values properly. The correct values were x_beam 159.353, y_beam 155.063 for DENZO/HKL2000 (Otwinowski \& Minor, 1997), BEAM 159.301 155.011 for MOSFLM (Leslie \& Powell, 2007), ORGX $=1512.73$ ORGY 1554.57 for $X D S$ (Kabsch, 2010) and origin= $-155.063,159.356,-250$ for cctbx/DIALS (GrosseKunstleve et al., 2002; Winter et al., 2018). Note that in addition to the $x-y$ flip between the $A D X V$ and MOSFLM/ $H K L-2000$ conventions, there is a half-pixel difference between the conventions of MOSFLM and HKL-2000 and a one-pixel difference between the MOSFLM and XDS conventions. Also, the $X D S$ and DIALS conventions do not use the beam itself as a reference point, so the values provided above are appropriate only when other program settings declare the detector plane to be perfectly orthogonal to the incident beam. This is usually the case at the start of processing, but refinement of the detector tilt will change these origin values. Detector tilts were simulated but were not included in the image header, specifically $0.365708^{\circ}$ forward detector tilt, $0.1145^{\circ}$ detector twist and $-0.140959^{\circ}$ detector rotation about the beam (CCOMEGA), as defined in the MOSFLM convention (Leslie \& Powell, 2007), and finally $0.0951363^{\circ}$ rotation of the spindle about the vertical axis away from normal to the beam. Although these numbers have many decimal places, they are the exact values that were fed into the simulation.

A total of 100 random orientation matrices with no orientation bias were pre-generated and used to create 100 simulated runs of 15 images each. Each run, or 'wedge', began with a new, fresh crystal that was assigned a cube shape with edge 
Table 1

Simulated crystal volumes $\left(\mu \mathrm{m}^{3}\right)$.

The true scale factor of the spots from each simulated data set is directly proportional to the simulated crystal volume, which was chosen randomly for each crystal. The actual values used in the simulation are listed here and may be used to check the accuracy of scaling programs as in Section 3.2 because no other variables such as the X-ray beam flux or even the structure factors were varied from crystal to crystal. The only remaining correction after this is the resolution-dependent scale factor of the simulated radiation damage described in Section 3.3.

\begin{tabular}{|c|c|c|c|c|c|c|c|c|c|}
\hline Crystal & Volume & Crystal & Volume & Crystal & Volume & Crystal & Volume & Crystal & Volume \\
\hline 001 & 225 & 021 & 139 & 041 & 132 & 061 & 50.3 & 081 & 105 \\
\hline 002 & 56.3 & 022 & 232 & 042 & 234 & 062 & 99.5 & 082 & 230 \\
\hline 003 & 63.9 & 023 & 155 & 043 & 46.9 & 063 & 196 & 083 & 171 \\
\hline 004 & 220 & 024 & 114 & 044 & 75.9 & 064 & 102 & 084 & 122 \\
\hline 005 & 186 & 025 & 38.4 & 045 & 51.6 & 065 & 229 & 085 & 56.8 \\
\hline 006 & 89.2 & 026 & 155 & 046 & 89.1 & 066 & 161 & 086 & 90.5 \\
\hline 007 & 52.2 & 027 & 46.7 & 047 & 230 & 067 & 72.4 & 087 & 90.2 \\
\hline 008 & 249 & 028 & 60.7 & 048 & 56.7 & 068 & 14.5 & 088 & 171 \\
\hline 009 & 185 & 029 & 70.7 & 049 & 97.8 & 069 & 131 & 089 & 186 \\
\hline 010 & 110 & 030 & 166 & 050 & 153 & 070 & 37.5 & 090 & 128 \\
\hline 011 & 166 & 031 & 143 & 051 & 237 & 071 & 207 & 091 & 42.2 \\
\hline 012 & 121 & 032 & 132 & 052 & 87.4 & 072 & 159 & 092 & 295 \\
\hline 013 & 160 & 033 & 213 & 053 & 130 & 073 & 88.4 & 093 & 240 \\
\hline 014 & 60.4 & 034 & 27.8 & 054 & 128 & 074 & 60.2 & 094 & 148 \\
\hline 015 & 189 & 035 & 210 & 055 & 86.4 & 075 & 190 & 095 & 51.5 \\
\hline 016 & 39.4 & 036 & 100 & 056 & 127 & 076 & 39.2 & 096 & 134 \\
\hline 017 & 47.6 & 037 & 12.5 & 057 & 52.8 & 077 & 186 & 097 & 46.3 \\
\hline 018 & 123 & 038 & 228 & 058 & 104 & 078 & 78.5 & 098 & 15.8 \\
\hline 019 & 277 & 039 & 210 & 059 & 146 & 079 & 108 & 099 & 201 \\
\hline 020 & 71.4 & 040 & 83.7 & 060 & 102 & 080 & 31.2 & 100 & 111 \\
\hline
\end{tabular}

dimension selected randomly about a $5 \mu \mathrm{m}$ average value and $1 \mu \mathrm{m}$ r.m.s. variation. Crystals larger than $6 \mu \mathrm{m}$ were cut off by the $6 \mu \mathrm{m}$ wide square beam. Although misalignment of the crystal with the X-ray beam was not explicitly modeled here, all misalignment does is reduce the illuminated volume, so the variability in crystal size modeled here can equally well be treated as crystal-to-crystal size variation or as same-size crystals with different degrees of misalignment. The only caveat to the latter is that this illuminated volume did not change with rotation, which keeps the ground-truth scale factor simple. The final illuminated volumes are listed in Table 1.

The X-ray beam was made to have a flux of $1 \times$ $10^{12}$ photons $^{-1}$ into a $6 \mu \mathrm{m}$ wide flat-top profile. The perimage exposure time was $1 \mathrm{~s}$ and $\Delta \Phi=1^{\circ}$. Shutter jitter was set to $2 \times 10^{-3}$ s r.m.s. in the starting and ending $\Phi$ values of each image, while beam flicker was taken to be $0.15 \% \mathrm{~Hz}^{-1 / 2}$ and implemented in ten steps per second. Beam divergence was set to $0.115 \times 0.0172^{\circ}$ (horizontal $\times$ vertical). These are typical measured properties of beamline 8.3.1 at the Advanced Light Source (MacDowell et al., 2004). Spectral dispersion, however, was set to $0.3 \%$ instead of the $0.014 \%$ measured from the $\mathrm{Si}(111)$ monochromator in order to mimic isotropic unit-cell variations in the sample (Nave, 1998). The mosaic spread was set to be a uniform disk of sub-crystal orientations with diameter $0.23^{\circ}$.

The X-ray background was also rendered on an absolute scale using realistic thicknesses of the materials in the beam: $20 \mathrm{~mm}$ of helium gas between the collimator and beam stop, and $5 \mu \mathrm{m}$ of liquid water and $4 \mu \mathrm{m}$ of Paratone-N oil in the beam path. Compton and diffuse scatter from the crystal lattice itself were computed based on the size and the composition of the macromolecule as described in the supplementary materials of Holton et al. (2014). Briefly, at the resolution where the Bragg spots fade into the background this diffuse component of the background converges to the same level as expected from all of the atoms in the protein crystal scattering independently, as if they were a gas.

\subsection{Simulated radiation-damage model}

Radiation damage was simulated in MLFSOM (Holton et $a l ., 2014)$ with only a simple, resolution-dependent exponential decay of spot intensities with dose using equation (13) from Holton \& Frankel (2010),

$$
I=I_{\mathrm{ND}} \exp \left[-\ln (2) \frac{D}{H d}\right],
$$

where $I_{\mathrm{ND}}$ is the intensity that would be observed in the absence of radiation damage, $I$ is the spot intensity at dose $D$ (MGy), $d$ is the resolution of the spot $(\AA)$ and $H$ is the $10 \mathrm{MGy} \AA^{-1}$ resolution dependence of the maximum tolerable dose estimated by Howells et al. (2009). For example, spots in the simulation at $2 \AA$ resolution were made to fade exponentially with dose, reaching half of $I_{\mathrm{ND}}$ after $20 \mathrm{MGy}$, and spots at $3.5 \AA$ resolution faded by half at $35 \mathrm{MGy}$. The dose was calculated assuming that the crystal was bathed in a flat-top beam using the formula 2000 photons $\mu \mathrm{m}^{-2} \mathrm{MGy}^{-1}$ from Holton (2009). This puts the first image at 13.9 MGy (see Fig. 1), and it should be noted that this end-of-image dose was used for the average dose of the entire image. No attempt was made to average over sub-image decay for this simulation, and the result was that the decay curve appears to be a perfect exponential offset in dose by half an image. Non-isomorphism 
owing to radiation damage was not simulated, and except for the simple exponential spot fading described above no variation in structure factors or unit cell with dose was employed. In fact, the unit-cell and structure-factor table was identical for all 100 simulated crystals, making this a case of perfect isomorphism. The reason for these unrealistically perfect damage and isomorphism models was to simplify the estimation of the errors in the cell and damage model introduced by the simulated noise as well as the data-processing algorithms themselves.

It is noteworthy that although (2) is consistent with 13 distinct studies of crystals and single particles using both $\mathrm{X}$-rays and electrons surveyed by Howells et al. (2009) over a resolution range of 2-600 $\AA$, it is not equivalent to a $B$ factor that increases with dose. This is incongruous with popular scaling programs, which use a quadratic ( $B$ factor) rather than a linear (2) resolution dependence for spot fading (Blake \& Phillips, 1962; Evans, 2006). Borek et al. (2013) describe one exception using SCALEPACK, but this non-Gaussian scaling option was only tested at low doses and is not the default. This damage model is therefore an example of a systematic error between the simulation and the internal models of scaling programs. These differences are detailed in Section 3.3, but it should be noted that the systematic error between reality and either of these decay models is no doubt even more complex. In this work, the average trend of spot fading versus resolution was used as the sole manifestation of radiation damage.

\section{Results and discussion}

In order to demonstrate the utility of this challenge, some discussion of the difficulties encountered when trying to solve the structure using MOSFLM (Leslie \& Powell, 2007), LABELIT (Sauter \& Poon, 2010), HKL-2000 (Otwinowski \&
Minor, 1997), XDS/XSCALE (Kabsch, 2010), DIALS (Winter et al., 2018), PHENIX (Adams et al., 2010), the CCP4 suite (Winn, 2003) and BLEND (Foadi et al., 2013) is provided here. Specific bugs and program-to-program differences will not be detailed here as software is continuously improving and contemporary shortcomings have little archival value, but the algorithmic challenge of simultaneous speed and robustness will be evaluated. The performance of particular programs with this data set is best described by their authors, such as Gildea \& Winter (2018).

\subsection{Automatic indexing}

Despite the high degree of similarity between these 100 simulated crystals, automated indexing was not always successful. Depending on the software used, the choice of images and the settings for spot picking and cell restraints, failures ranged from exiting with an error message to confidently arriving at an incorrect Niggli cell, usually with one or more of the primitive cell dimensions doubled. This type of mis-indexing could not be corrected by downstream reindexing programs such as POINTLESS (Evans, 2006, 2011), and thus represents a significant barrier to including these particular wedges.

A naïve user might even mistake such mis-indexing for evidence of variations in crystal habit, so it is important to note here that there was no difference in quality between any of these simulated crystals. All wedges had the same resolution and the same decay rate and were perfectly isomorphous. The true unit cells were all identical as well, which allowed calibration of the influence of random noise on cell refinement. Clustering the refined unit cells using BLEND (Foadi et al., 2013) demonstrated that an LCV of $\sim 1 \%$ does not necessarily imply non-isomorphism, and that even random

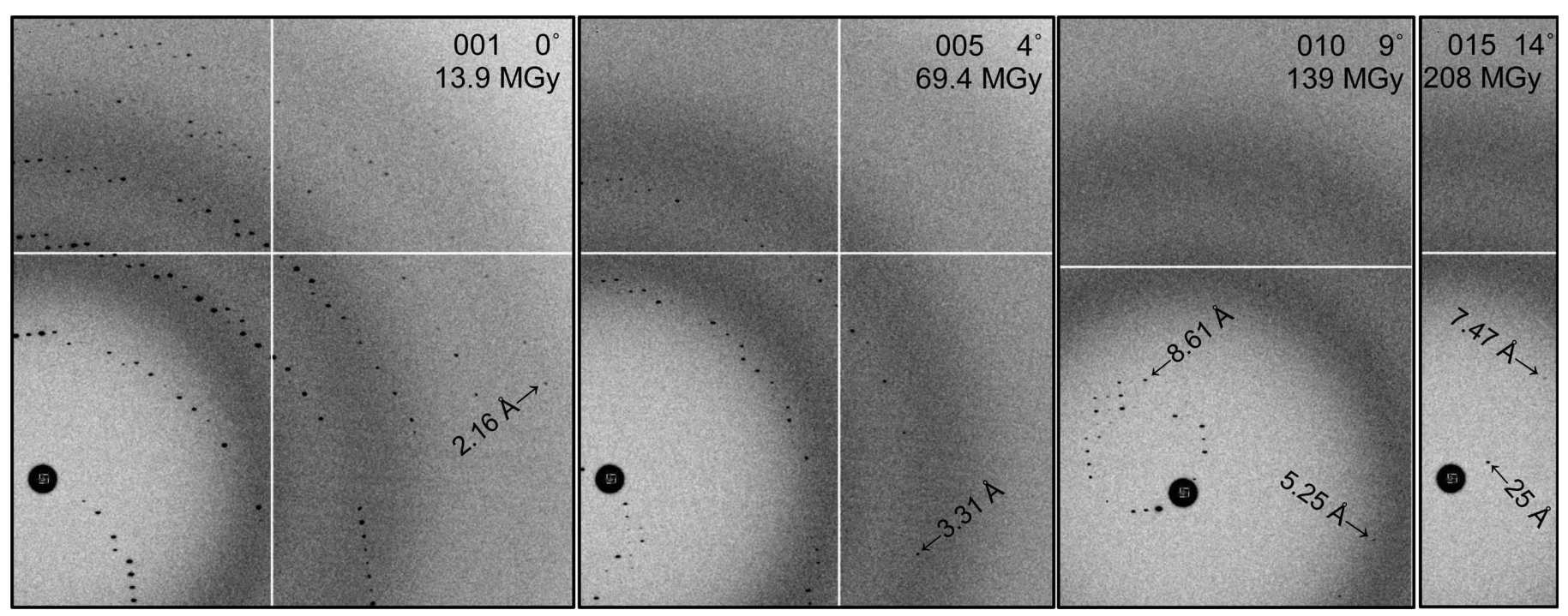

Figure 1

Enlarged sections of diffraction patterns from simulated crystal 016. Six lunes are apparent on image 001, but indexing this wedge still proved problematic. The resolution-dependent exponential fading of spots with dose is exemplified by the rapid loss of high-angle data and the relative persistence of low-angle features. Despite perfect isomorphism, images 004 and higher degraded the overall anomalous signal and images 002 and higher degraded the overall resolution of the final data set. 
relationships still produce a dendrogram with major and minor branching (Fig. 2).

Aside from orientation, the only major difference between the simulated crystals was the illuminated volume, which varied over a factor of 24 (Table 1). However, neither the smallest (037) nor the largest (092) simulated crystal had indexing problems. The most problematic crystals were 016, 064, 065, 086 and 095, all of which have one reciprocal-cell axis close to parallel to the incident beam. This situation can cause problems in indexing because the information about the cell axis near the beam is maximally distorted by the Ewald sphere and may even be missing entirely if the crystal diffracts poorly and produces only one lune (Brewster et al., 2018). However, all of these problematic wedges diffracted to $1.8 \AA$ resolution and displayed 3-6 clear lunes, so the reason for these failures is not immediately clear. In addition to these five problem crystals, four others, 051, 054, 062 and 063, failed with most combinations of images but not all, and 11 more, 004, 006, 010, 019, 065, 068, 086, 094, 097 and 098, usually succeeded but failed with at least one combination of images. Since the major difference was the crystal orientation, the indexing algorithm itself may be considered to be a source of orientational bias in

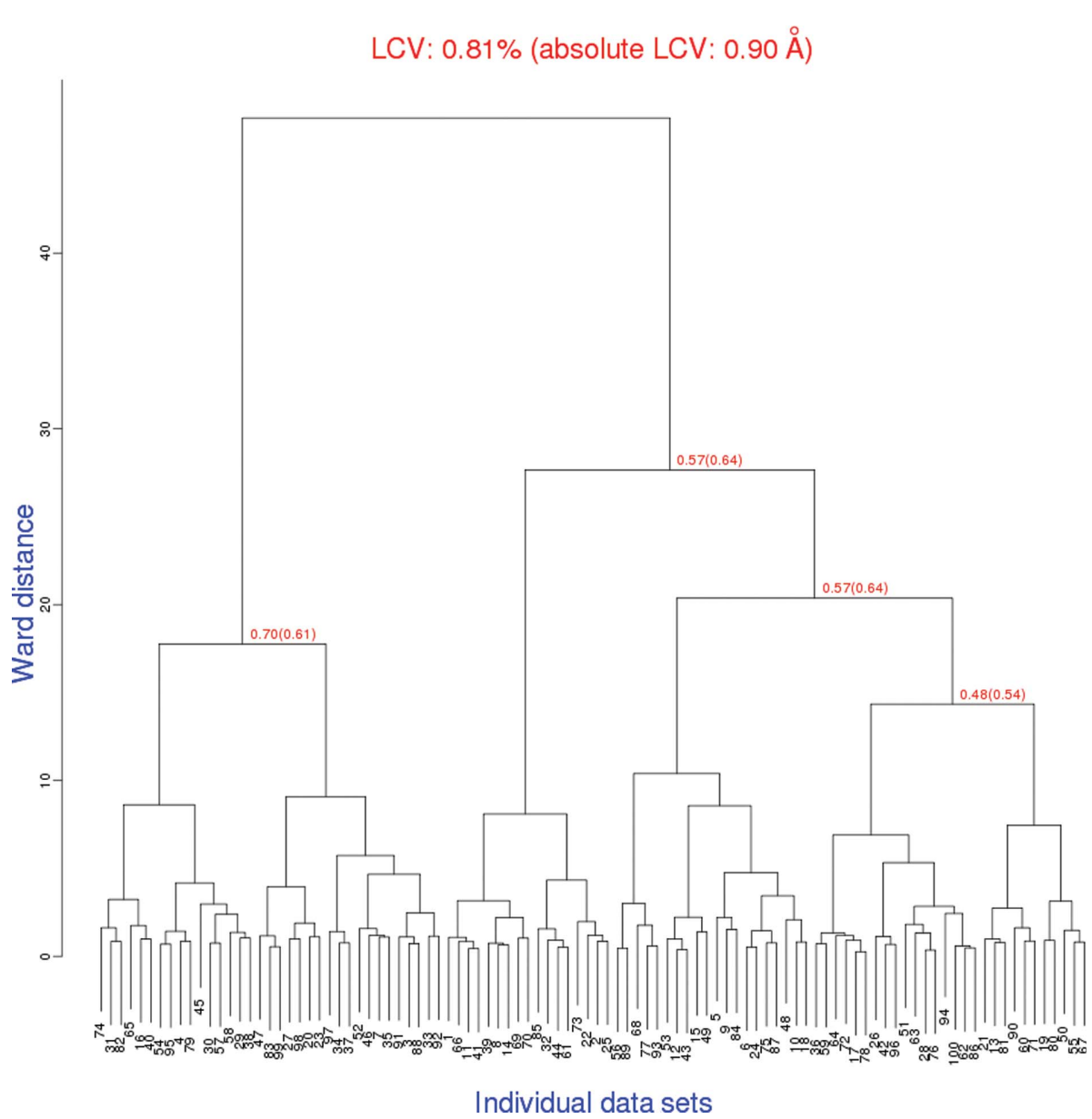

Figure 2

BLEND (Foadi et al., 2013) dendrogram of unit cells obtained from XDS (Kabsch, 2010) processing. Although the clustering suggests groups of related crystals, the true underlying unit cells and structure factors were identical for all 100 wedges. The unit-cell variation shown here is therefore entirely owing to the impact of random noise on indexing and cell refinement. multi-crystal data, even if the true orientation distribution is isotropic.

In general the fastest programs had the highest failure rates, whereas more complex algorithms took longer but arrived at the correct Niggli cell more reliably, such as that of Sauter \& Zwart (2009). Execution times varied from 0.3 to $9 \mathrm{~s}$ across the programs tested, so the tradeoff between speed and robustness is significant. However, these same more complex algorithms were vulnerable to other considerations, such as weak images. For example, LABELIT indexing with images 1 and 15 failed in 78/100 cases, but the same program given images 1 and 4 found the correct lattice for 100/100 cases. A combinatorial approach scanning over image selection and other program settings would no doubt be most robust, but would also consume the most computing resources.

Automatic space-group determination also had its flaws. Essentially all indexing software tested arrived at a tetragonal solution, which is not intrinsically problematic until after the merging step, but the completeness of any given single wedge was so low $(\sim 10 \%)$ that few symmetry operators could be eliminated for any particular wedge taken in isolation. For example, POINTLESS (Evans, 2006, 2011) assigned most of the 100 simulated crystals to space groups $P 1(35 \%)$ or $P 2(23 \%)$, while some were assigned to $P 222$ (11\%), C2 $(12 \%)$ or $P 422(9 \%)$ and in rare cases to $C 222$ or $P 4$, indicating that the true space group is not obvious from the primary data. It is commonplace to assign the highest symmetry possible during processing in order to maximize the completeness of each wedge and therefore the overlap with other wedges to make cross-crystal scaling simpler and more robust. However, pursuing this strategy invariably ended with what appeared to be extremely noisy data that did not merge well and appeared to be twinned. The final $R$ factor between $F_{\text {sim }}$ and $F_{\text {right }}$ was $53 \%$. The most robust strategy and unfortunately the most computationally intensive remained independently pursuing processing, scaling, merging and combining data in all possible point groups separately, and in addition scanning over all possible radiation-damage cutoffs. This is a large number of combinations, but the correct point group (222) and cutoff (three images) were only clear when both were applied at the same time.

One trick that proved to be helpful in solving this data set (Diederichs, 2016; Gildea \& Winter, 2018) is to initially drop all symmetry to $P 1$. This avoids overestimation of symmetry and worked well for the present challenge 
data. However, it is expected that for real-world cases that have poorer resolution and more incomplete wedges working in $P 1$ will be limiting. For example, cell refinement is less stable when the lattice is completely unrestrained. The connectivity between wedges is also minimized by comparing them in $P 1$ because many observations that would be symmetry-equivalent in the true crystal symmetry are not equivalent in $P 1$. This lack of overlap makes resolving the indexing ambiguity harder or even impossible in the limit of sparse data from few crystals. It is expected that finding a way to reliably identify and take advantage of the internal symmetry within each wedge will be a valuable future development.

\subsection{Cheating}

In order to demonstrate an ideal solution to this challenge, the simulated data were processed using $F_{\text {right }}$ as a reference for the unit cell and structure factors. This eliminated any indexing ambiguity. The unit cell and space group were also fixed to the correct values during indexing, refinement and integration in MOSFLM (Leslie \& Powell, 2007). The best radiation-damage cutoff was determined empirically by scaling and merging all 100 correctly indexed wedges together with POINTLESS/AIMLESS (Evans, 2011) and comparing the final merged structure factors with $F_{\text {right }}$.

The optimum cutoff to optimize weak, high-resolution data was to use only the first image, as shown in Fig. 3. Although scaling programs such as AIMLESS take a 'run' of images, for this case each run started and ended with image ' 1 ', a strategy that also eliminates all partially recorded reflections. Using just the first image from each wedge also minimized the

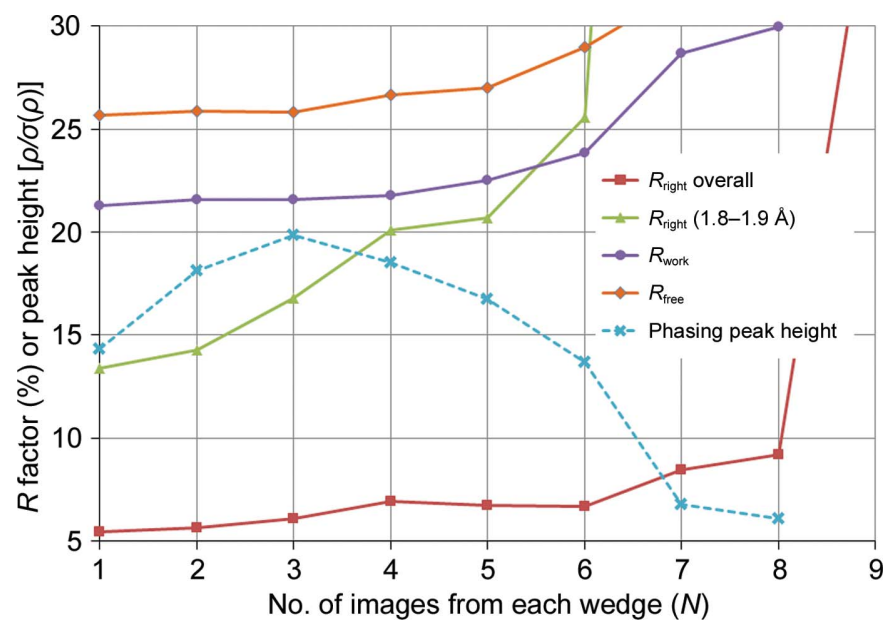

Figure 3

Graph of the relative error $\left(R_{\text {right }}\right)$ between the correct structure factor $\left(F_{\text {right }}\right)$ and the structure factor obtained from scaling and merging the first $N$ images from all 100 simulated crystals $\left(F_{\text {sim }}\right)$. Also shown are $R_{\text {work }}$ and $R_{\text {free }}$ from refinement to convergence of the correct starting model against $F_{\text {sim }}$ from $N$-image data. Despite perfect isomorphism, fewer images resulted in better agreement. The $y$ axis also represents the maximum peak height found in the phased anomalous difference Fourier (dashed line). Phases were obtained by removing all Se atoms before refining to convergence against $F_{\text {sim. }}$. The phasing signal is maximized at $N=3$. overall $R_{\text {work }}$ to $21.3 \%$ and $R_{\text {free }}$ to $25.7 \%$ after refining the selenated reference model PDB entry $1 \mathrm{~g} 1 \mathrm{c}$ to convergence with REFMAC (Murshudov et al., 2011). This is most likely because the increase in $R_{\text {right }}$ with increasing $N$ shown in Fig. 3 was due to unstable scaling. After correcting for the known crystal volumes (Table 1), the r.m.s. variation in the scale factor assigned to spots in the $1.8-1.9 \AA$ bin was $18 \%$ for $N=5$ but was only $1.4 \%$ at $N=1$. This was almost entirely owing to variation in the scaling $B$ factor, which was actually invariant from crystal to crystal in the simulation. The reason for this instability is suspected to be the incongruence of radiationdamage models detailed in Section 3.3.

The optimum anomalous signal was attained using the first three images of each wedge (Fig. 3), and structure solution was straightforward using automated phasing pipelines, much as reported by Gildea \& Winter (2018). Structure solution was also possible with fewer data, down to crystals 001-042, with SHELXC/D/E (Sheldrick, 2015; Usón \& Sheldrick, 2018), indicating the threshold of solvability with ideal data processing. All four correct selenium sites, as evaluated with phenix.emma, were found with SHELXD using as few data as crystals 001-029 with $\mathrm{CC}_{\text {all }} / \mathrm{CC}_{\text {weak }}$ at $30 / 20 \%$. Applying a further cheat of providing SHELXE with the correct selenium and sulfur sites allowed the application of the twofold NCS, making structure solution possible down to crystals 001-036. Better results are expected with further cheats, such as directly correcting the exponential spot decay, but this was not attempted in the present work. Nondefault parameters that were necessary for success were instructing SHELXD to find four sites with a resolution cutoff of $3.5 \AA$ and MIND -3.5 . For SHELXE using the correct sites the required options were -s $0.53-\mathrm{n} 2-\mathrm{a} 100-\mathrm{w} 0.3-\mathrm{F} 0.7-\mathrm{t} 5-\mathrm{L} 1-\mathrm{B} 3$. Using the SHELXD sites, solution was possible down to crystals 001-040 with the options -s0.53-a100-t1 -B3-L1. No parameters could be found to solve the structure using crystals 001-035, despite a systematic search over $>9000$ distinct sets.

A script provided as supporting information reproduces the solutions described above, but it should be noted that near the threshold any protocol will be fragile. Changing any parameter, such as using a processing program other than MOSFLM, or even using different CPU types, could make or break the solution. As crystallographic software evolves these sensitivities are expected to disappear and perhaps new ones will manifest. It is therefore recommended to start with the robust case of merging 100 crystals and then to start dropping crystals from the tail end until the limitation of the pipeline of interest is found. It is at this threshold that the vulnerabilities of any given algorithm are most easily detected and corrected.

\subsection{Resolution dependence of radiation damage}

The non-Gaussian nature of the damage model used in this simulation was unexpectedly detrimental to contemporary scaling procedures, so here we shall place this empirical decay equation into context with the conventional scale-and- $B$ factor model. It is instructive to recast (2) in the same form as a $B$ factor $\left[\exp \left(-B s^{2}\right)\right]$ by defining $A=\ln (2) D / H$, substituting 
the resolution $d$ with the reciprocal scattering-vector length $s=(2 d)^{-1}$ and converting intensities $(I)$ to structure factors $(F)$ by taking the square root of both sides. The factor of two in the switch from $d$ to $s$ is canceled by the switch from intensities to structure factors, and we arrive at

$$
F=F_{\mathrm{ND}} \exp (-A s),
$$

where $F_{\mathrm{ND}}$ is the structure factor of the damage-free unit cell. This rearranged spot-fading formula immediately suggests a Taylor expansion in the exponent, demonstrating the relationship between $A$ and $B$, and perhaps additional factors such as $C$. Let us briefly entertain this formalism, and write

$$
F=F_{\mathrm{ND}} \exp \left(-A s-B s^{2}-C s^{3}\right),
$$

where $B$ is the usual $B$ factor $\left(8 \pi^{2}\left\langle u_{x}^{2}\right\rangle\right)$, in which $u_{x}$ is the component of the Gaussian-distributed atomic displacement vector $\mathbf{u}$ in the direction normal to the Bragg plane and \langle\rangle denotes the mean over all atoms. Similarly, $A=2 \pi w_{\text {fhm }}$, where $w_{\text {fhm }}$ is the full-width at half-maximum of atomic displacements taken from the multivariate Cauchy-Lorentz distribution,

$$
P(\mathbf{u})=\frac{8}{\pi^{2} w_{\text {fhm }}^{3}}\left[1+\left(\frac{2|\mathbf{u}|}{w_{\text {fhm }}}\right)^{2}\right]^{-1},
$$

where $P(\mathbf{u})$ is the normalized probability of atomic displacement vector $\mathbf{u}$ and $\|$ denotes the vector magnitude (in $\AA$ ). This distribution resembles a Gaussian but has heavier tails, indicating a much higher ratio of large-scale to small-scale movements than would be expected from a Gaussian distribution. Generating this distribution must be performed with care because one cannot simply apply three independent displacements along $x, y$ and $z$, as this creates a highly anisotropic three-dimensional histogram. Rather, a random direction for $\mathbf{u}$ must first be chosen and (5) applied along its axis.

It was argued by Debye (1914) that all terms except $B s^{2}$ in (4) vanish when averaged over the large number of atoms in the crystal (equation I.26 in James, 1962), but this is only the case when the distribution of atomic displacements converges to a Gaussian via the central limit theorem. There are random distributions that do not obey the central limit theorem, and the Cauchy-Lorentz distribution is one example. In fact, combinations of Cauchy-Lorentz deviates always converge to another Cauchy-Lorentz distribution, forming an analogous but distinct version of the central limit theorem.

Strictly speaking, the falloff of intensity with resolution owing to any distribution of atomic displacements is the Fourier transform of that distribution. The Fourier transform of a Gaussian atomic displacement distribution is another Gaussian (the $B$ factor), and the Fourier transform of a Cauchy-Lorentz distribution is an exponential in reciprocal space, as in (3). If the manifestation of radiation damage is a $B$ factor that increases linearly with dose, then the spot-fading half-dose would be related to the square of resolution, not linearly. The observation by Howells of a linear relationship between resolution and spot-fading half-dose therefore implies a direct proportionality between dose and the width of the distribution of atomic displacements,

$$
w_{\text {fhm }}=\frac{D \ln (2)}{2 \pi H},
$$

where $D$ is the dose in MGy, $\ln (2)$ is the natural $\log$ of 2 and $H$ is the $10 \mathrm{MGy}^{-1}$ trend observed by Howells. Here, we use the full-width at half-maximum to describe the CauchyLorentz histogram rather than the r.m.s. variation because the r.m.s. variation of a Cauchy-Lorentz distribution is undefined, as is its mean. A physically reasonable explanation for the departure from Gaussian-distributed atomic displacements may be that large enough displacements require neighboring atoms to move out of the way, creating additional large u vectors of similar magnitude and direction, and leading to a higher than 'normally' expected population of large $\mathbf{u}$ vectors. Cracking and slipping of lattice fragments relative to each other may be examples of such concerted movements.

As a historical aside, the appearance of the letter $B$ as the second term in (4) invites speculation that it is the origin for the choice of the letter $B$ to indicate the Debye-Waller-Ott factor, and therefore a natural place for $A$ and $C$ factors. This is not actually the case. The first use of $B$ to describe Debye's disorder parameter appeared in Bragg (1914), and therein the letter $A$ was used to encapsulate the overall scale factor, which is in no way analogous to the Cauchy-Lorentz term in (4). What is more, the $C$ factor does not relate to any physically reasonable distribution because its corresponding real-space displacement histogram has negative population values, and probabilities cannot be negative. So, although (4) resembles a Taylor expansion in the exponent, only the first two terms $A$ and $B$ correspond to physically plausible distributions.

\section{Conclusions}

The challenges to macromolecular structure determination using data from a large number of small crystals lie primarily in the combinatorial nature of the data analysis. Recent landmark achievements such as those reported by Brehm \& Diederichs (2014), Liu \& Spence (2014), Gildea \& Winter (2018), Diederichs $(2016,2017)$ and, in this issue, Foos et al. (2019) represent important mathematical advances in handing this problem and significant practical progress towards solving the present challenge. The indexing-ambiguity problem itself may now be regarded as solved, with the proviso that current approaches are still vulnerable to incorrect lattice assignment, such as cell doubling, and radiation-damage cutoffs during processing. These choices are still up to the user, and since the correct choice is generally not clear until the structure has been solved, the only robust strategy remains an exhaustive evaluation of all possible lattice-type and damage-cutoff options. By 'cheating' this work was able to solve the challenge structure using only the first 36 crystals of the 100 presented, and further work that can approach or surpass this number without cheating will directly translate to real-world projects finishing earlier and using fewer difficult-to-produce isomorphous crystalline samples. 
It is tempting to suggest overcoming indexing problems by using a pair of orthogonal alignment shots prior to data collection, but since only the first three images appear to be useful before the data quality degrades this strategy is not recommended. Lowering the exposure time and covering more of reciprocal space with the same dose is expected to improve the indexing performance, but this strategy is not applicable to the problem of serial crystallography (Wiedorn et al., 2018; Chapman et al., 2011), where particularly at XFEL sources only one image is available from each sample. The limit of how weak individual images can be before resolution begins to degrade will be the subject of a future challenge, but recent results have shown that this limit can be quite low (Lan et al., 2018; Parkhurst et al., 2016). It is further expected that as radiation-damage processes become better understood and correctable including more images will improve data quality rather than degrade it.

The challenge proposed here is to beat the 36-crystal limit and solve this structure by anomalous phasing without 'cheating' in any way. In the real world a reference data set may not be available or appropriate if the crystals are not very reproducible. Realistic solutions to the indexing ambiguity must also be able to handle the inaccurate first-pass symmetry determination that is inherent to highly incomplete data sets, and automatic radiation-damage cutoffs must become more reliable to be of practical use.

\section{Acknowledgements}

I would like to thank Drs Christine Gee and Nicholas Sauter for extremely helpful discussions of this manuscript, and George Sheldrick and Isabel Usón for their advice with SHELXE. Images have been deposited in the IRRMC at https://proteindiffraction.org/ (DOI link: https://doi.org/ 10.18430/microfocus_challenge_2011), and are also available at http://bl831.als.lbl.gov/ jamesh/challenge/microfocus/.

\section{Funding information}

This work was supported by grants from the National Institutes of Health (GM124149, GM124169, GM103393, GM082250), The National Science Foundation (DBI-1625906), UC Multicampus Research Projects and Initiatives (award No. MR-15-328599) and the US Department of Energy under contract Nos. DE-AC02-05CH11231 at Lawrence Berkeley National Laboratory and DE-AC02-76SF00515 at SLAC National Accelerator Laboratory.

\section{References}

Adams, P. D., Afonine, P. V., Bunkóczi, G., Chen, V. B., Davis, I. W., Echols, N., Headd, J. J., Hung, L.-W., Kapral, G. J., GrosseKunstleve, R. W., McCoy, A. J., Moriarty, N. W., Oeffner, R., Read, R. J., Richardson, D. C., Richardson, J. S., Terwilliger, T. C. \& Zwart, P. H. (2010). Acta Cryst. D66, 213-221.

Arvai, A. (2012). ADXV-A Program to Display X-ray Diffraction Images. https://www.scripps.edu/tainer/arvai/adxv.html.

Bedem, H. van den, Dhanik, A., Latombe, J.-C. \& Deacon, A. M. (2009). Acta Cryst. D65, 1107-1117.
Berman, H. M., Battistuz, T., Bhat, T. N., Bluhm, W. F., Bourne, P. E., Burkhardt, K., Feng, Z., Gilliland, G. L., Iype, L., Jain, S., Fagan, P., Marvin, J., Padilla, D., Ravichandran, V., Schneider, B., Thanki, N., Weissig, H., Westbrook, J. D. \& Zardecki, C. (2002). Acta Cryst. D58, 899-907.

Blake, C. C. F. \& Phillips, D. C. (1962). Biological Effects of Ionizing radiation at the Molecular Level, pp. 183-191. Vienna: IAEA.

Borek, D., Dauter, Z. \& Otwinowski, Z. (2013). J. Synchrotron Rad. 20, 37-48.

Bragg, W. H. (1914). Lond. Edinb. Dubl. Philos. Mag. J. Sci. 27, 881899.

Brehm, W. \& Diederichs, K. (2014). Acta Cryst. D70, 101-109.

Brewster, A. S., Waterman, D. G., Parkhurst, J. M., Gildea, R. J., Young, I. D., O'Riordan, L. J., Yano, J., Winter, G., Evans, G. \& Sauter, N. K. (2018). Acta Cryst. D74, 877-894.

Chapman, H. N., Fromme, P., Barty, A., White, T. A., Kirian, R. A., Aquila, A., Hunter, M. S., Schulz, J., DePonte, D. P., Weierstall, U., Doak, R. B., Maia, F. R. N. C., Martin, A. V., Schlichting, I., Lomb, L., Coppola, N., Shoeman, R. L., Epp, S. W., Hartmann, R., Rolles, D., Rudenko, A., Foucar, L., Kimmel, N., Weidenspointner, G., Holl, P., Liang, M., Barthelmess, M., Caleman, C., Boutet, S., Bogan, M. J., Krzywinski, J., Bostedt, C., Bajt, S., Gumprecht, L., Rudek, B., Erk, B., Schmidt, C., Hömke, A., Reich, C., Pietschner, D., Strüder, L., Hauser, G., Gorke, H., Ullrich, J., Herrmann, S., Schaller, G., Schopper, F., Soltau, H., Kühnel, K.-U., Messerschmidt, M., Bozek, J. D., Hau-Riege, S. P., Frank, M., Hampton, C. Y., Sierra, R. G., Starodub, D., Williams, G. J., Hajdu, J., Timneanu, N., Seibert, M. M., Andreasson, J., Rocker, A., Jönsson, O., Svenda, M., Stern, S., Nass, K., Andritschke, R., Schröter, C.-D., Krasniqi, F., Bott, M., Schmidt, K. E., Wang, X., Grotjohann, I., Holton, J. M., Barends, T. R. M., Neutze, R., Marchesini, S., Fromme, R., Schorb, S., Rupp, D., Adolph, M., Gorkhover, T., Andersson, I., Hirsemann, H., Potdevin, G., Graafsma, H., Nilsson, B. \& Spence, J. C. H. (2011). Nature (London), 470, 73-77.

Debye, P. J. W. (1914). Ann. Phys. 348, 49-92.

Diederichs, K. (2016). Serial Synchrotron Crystallography: Data Processing. https://strucbio.biologie.uni-konstanz.de/xdswiki/ index.php/SSX.

Diederichs, K. (2017). Acta Cryst. D73, 286-293.

Evans, G., Axford, D., Waterman, D. \& Owen, R. L. (2011). Crystallogr. Rev. 17, 105-142.

Evans, P. (2006). Acta Cryst. D62, 72-82.

Evans, P. R. (2011). Acta Cryst. D67, 282-292.

Foadi, J., Aller, P., Alguel, Y., Cameron, A., Axford, D., Owen, R. L., Armour, W., Waterman, D. G., Iwata, S. \& Evans, G. (2013). Acta Cryst. D69, 1617-1632.

Foos, N., Cianci, M. \& Nanao, M. H. (2019). Acta Cryst. D75, 200210.

Gildea, R. J. \& Winter, G. (2018). Acta Cryst. D74, 405-410.

Grabowski, M., Langner, K. M., Cymborowski, M., Porebski, P. J., Sroka, P., Zheng, H., Cooper, D. R., Zimmerman, M. D., Elsliger, M.-A., Burley, S. K. \& Minor, W. (2016). Acta Cryst. D72, 11811193.

Grosse-Kunstleve, R. W., Sauter, N. K., Moriarty, N. W. \& Adams, P. D. (2002). J. Appl. Cryst. 35, 126-136.

Gruner, S. M. (1989). Rev. Sci. Instrum. 60, 1545-1551.

Gruner, S. M., Tate, M. W. \& Eikenberry, E. F. (2002). Rev. Sci. Instrum. 73, 2815-2842.

Holton, J. M. (2009). J. Synchrotron Rad. 16, 133-142.

Holton, J. M., Classen, S., Frankel, K. A. \& Tainer, J. A. (2014). FEBS J. 281, 4046-4060.

Holton, J. M. \& Frankel, K. A. (2010). Acta Cryst. D66, 393-408.

Holton, J. M., Nielsen, C. \& Frankel, K. A. (2012). J. Synchrotron Rad. 19, 1006-1011.

Howells, M. R., Beetz, T., Chapman, H. N., Cui, C., Holton, J. M., Jacobsen, C. J., Kirz, J., Lima, E., Marchesini, S., Miao, H., Sayre, D., Shapiro, D. A., Spence, J. C. H. \& Starodub, D. (2009). J. Electron Spectrosc. Relat. Phenom. 170, 4-12. 
James, R. W. (1962). The Optical Principles of The Diffraction of $X$-rays. London: Bell.

Kabsch, W. (2010). Acta Cryst. D66, 125-132.

Lan, T.-Y., Wierman, J. L., Tate, M. W., Philipp, H. T., Martin-Garcia, J. M., Zhu, L., Kissick, D., Fromme, P., Fischetti, R. F., Liu, W., Elser, V. \& Gruner, S. M. (2018). IUCrJ, 5, 548-558.

Leslie, A. G. W. \& Powell, H. R. (2007). Evolving Methods for Macromolecular Crystallography, edited by R. Read \& J. Sussman, pp. 41-51. Dordrecht: Springer.

Liu, H. \& Spence, J. C. H. (2014). IUCrJ, 1, 393-401.

MacDowell, A. A., Celestre, R. S., Howells, M., McKinney, W., Krupnick, J., Cambie, D., Domning, E. E., Duarte, R. M., Kelez, N., Plate, D. W., Cork, C. W., Earnest, T. N., Dickert, J., Meigs, G., Ralston, C., Holton, J. M., Alber, T., Berger, J. M., Agard, D. A. \& Padmore, H. A. (2004). J. Synchrotron Rad. 11, 447-455.

Mayans, O., Wuerges, J., Canela, S., Gautel, M. \& Wilmanns, M. (2001). Structure, 9, 331-340.

McGill, K. J., Asadi, M., Karakasheva, M. T., Andrews, L. C. \& Bernstein, H. J. (2014). J. Appl. Cryst. 47, 360-364.

Morin, A., Eisenbraun, B., Key, J., Sanschagrin, P. C., Timony, M. A., Ottaviano, M. \& Sliz, P. (2013). Elife, 2, e01456.

Murshudov, G. N., Skubák, P., Lebedev, A. A., Pannu, N. S., Steiner, R. A., Nicholls, R. A., Winn, M. D., Long, F. \& Vagin, A. A. (2011). Acta Cryst. D67, 355-367.

Nave, C. (1998). Acta Cryst. D54, 848-853.

Otwinowski, Z. \& Minor, W. (1997). Methods Enzymol. 276, 307-326.

Parkhurst, J. M., Brewster, A. S., Fuentes-Montero, L., Waterman, D. G., Hattne, J., Ashton, A. W., Echols, N., Evans, G., Sauter, N. K. \&
Winter, G. (2014). J. Appl. Cryst. 47, 1459-1465.

Parkhurst, J. M., Winter, G., Waterman, D. G., Fuentes-Montero, L., Gildea, R. J., Murshudov, G. N. \& Evans, G. (2016). J. Appl. Cryst. 49, 1912-1921.

Sauter, N. K. \& Poon, B. K. (2010). J. Appl. Cryst. 43, 611-616.

Sauter, N. K. \& Zwart, P. H. (2009). Acta Cryst. D65, 553-559.

Sheldrick, G. M. (2015). Acta Cryst. C71, 3-8.

Simpkin, A. J., Simkovic, F., Thomas, J. M. H., Savko, M., Lebedev, A., Uski, V., Ballard, C., Wojdyr, M., Wu, R., Sanishvili, R., Xu, Y., Lisa, M.-N., Buschiazzo, A., Shepard, W., Rigden, D. J. \& Keegan, R. M. (2018). Acta Cryst. D74, 595-605.

Szebenyi, D. M. E., Arvai, A., Ealick, S., LaIuppa, J. M. \& Nielsen, C. (1997). J. Synchrotron Rad. 4, 128-135.

Usón, I. \& Sheldrick, G. M. (2018). Acta Cryst. D74, 106-116.

Waterman, D. \& Evans, G. (2010). J. Appl. Cryst. 43, 1356-1371.

Wiedorn, M. O., Awel, S., Morgan, A. J., Ayyer, K., Gevorkov, Y., Fleckenstein, H., Roth, N., Adriano, L., Bean, R., Beyerlein, K. R., Chen, J., Coe, J., Cruz-Mazo, F., Ekeberg, T., Graceffa, R., Heymann, M., Horke, D. A., Knoška, J., Mariani, V., Nazari, R., Oberthür, D., Samanta, A. K., Sierra, R. G., Stan, C. A., Yefanov, O., Rompotis, D., Correa, J., Erk, B., Treusch, R., Schulz, J., Hogue, B. G., Gañán-Calvo, A. M., Fromme, P., Küpper, J., Rode, A. V., Bajt, S., Kirian, R. A. \& Chapman, H. N. (2018). IUCrJ, 5, 574-584. Winn, M. D. (2003). J. Synchrotron Rad. 10, 23-25.

Winter, G., Waterman, D. G., Parkhurst, J. M., Brewster, A. S., Gildea, R. J., Gerstel, M., Fuentes-Montero, L., Vollmar, M., MichelsClark, T., Young, I. D., Sauter, N. K. \& Evans, G. (2018). Acta Cryst. D74, 85-97. 